\title{
Semi-annual variation of the geomagnetic field
}

\author{
S. R. C. Malin ${ }^{1}$, D. E. Winch ${ }^{2}$, and A. M. Işıkara ${ }^{1}$ \\ ${ }^{1}$ Bog̀aziçi University, Kandilli Observatory and Earthquake Research Institute, 81220, Çengelköy, Istanbul, Turkey \\ ${ }^{2}$ School of Mathematics and Statistics, University of Sydney, F07, Sydney, NSW 2006, Australia
}

(Received September 29, 1998; Revised March 23, 1999; Accepted April 21, 1999)

\begin{abstract}
Nighttime hourly mean values of $D, H$ and $Z$ (or $X, Y$ and $Z$ in a few cases) from 113 observatories for the interval 1964.0 to 1966.0 have been analyzed to determine the semi-annual variation. Results from the 84 observatories with dip latitudes between $\pm 60^{\circ}$ have been subjected to spherical harmonic analysis to determine the coefficients of the internal and external parts. Only those coefficients that are found to be significantly different from zero at the 5 per cent level have been included.

One of the main objectives is to obtain a reliable estimate, with confidence limits, of the internal/external ratio at a very low frequency for constraining estimates of the deep conductivity of the mantle. It is shown that a model that includes only the principal $P_{1}^{0}$ term can lead to a seriously misleading internal/external ratio.
\end{abstract}

\section{Introduction}

The solar-cycle, the annual and semi-annual variations are the longest period peaks of external origin in the geomagnetic spectrum. Thus they are of particular importance for placing constraints on estimates of the lower-mantle conductivity. Global models have been made of the 11-year and annual variations (e.g. Malin and Işıkara, 1976; Harwood and Malin, 1977), but modelling of the semi-annual variation has hitherto been confined to the principal $P_{1}^{0}$ term (e.g. Banks, 1972). The particular importance of the semi-annual variation is that, apart from the 27-day recurrence tendency and its harmonics, it is the only significant spectral peak between the daily (24-hour) and annual lines (see Black, 1970).

It is important to distinguish between the semi-annual variation of the field itself, considered here, and the more widely studied semi-annual variation of magnetic activity (McIntosh, 1959; Gupta and Chapman, 1967; Russell and McPherron, 1973). The two phenomena both have extrema near the equinoxes suggesting a common origin. There are several possible mechanisms. Since we use data for quietSun years and omit the 5 International Disturbed Days of each month, the contribution of magnetospheric compression is minimal. Also, by using only nighttime data, the effect of ionospheric currents is virtually eliminated. So the variation we are looking at here is likely to be due principally to semi-annual changes in strength or position of the ringcurrent. One possible mechanism is that more solar-wind ions are available for trapping when the Earth is at relatively high latitudes. The Earth is at high heliographic latitude $\left(>7.23^{\circ}\right)$ for the week centred on September 9 and is at low heliographic latitude $\left(<-7.23^{\circ}\right)$ for the week centred on March 7; both times precede the equinoxes by approximately two weeks. Another possibility is that the magnetosphere is

Copy right(C) The Society of Geomagnetism and Earth, Planetary and Space Sciences (SGEPSS); The Seismological Society of Japan; The Volcanological Society of Japan; The Geodetic Society of Japan; The Japanese Society for Planetary Sciences. more effective at trapping particles when it is symmetrical with respect to the Earth-Sun line, viz. at the equinoxes. The annual north-south migration of the ring-current postulated by Fukushima and Nagata (1968) and by Malin and Işıkara (1976) would cause it to pass directly over a low latitude observatory twice a year, resulting in a semi-annual variation. This effect has been investigated by McCreadie and Butcher (1993).

The purpose of the present study is threefold. Firstly, it presents the first detailed global model of semi-annual variation. Previous analyses have either been confined to a small number of observatories, with no attempt at global coverage, to investigate specific features (Campbell, 1980, 1981; Hibberd, 1985; Rangarajan and Bhargava, 1987; Butcher and Schlapp, 1992; Rastogi, 1993; Rastogi et al., 1994), or have been concerned with only one, or a very few spherical harmonics (McLeod, 1994). Secondly, we provide data which, in combination with other investigations, should help to elucidate at least the ring current part of the mechanism for the semi-annual variation. Thirdly, it provides data at an important frequency for use in estimating the conductivity of the mantle.

\section{The Data}

The data are hourly mean values of the magnetic elements of declination, $D$, horizontal intensity, $H$, and vertical intensity, $Z$, (or, in a few cases, north intensity, $X$, east intensity $Y$, and $Z$ ) from magnetic observatories. They have been compiled and put into machine-readable form by D. E. Winch, with some corrections by U. Schmucker, and further corrections to Pilar and Teoloyucan. The data cover the interval from 1964.0 to 1966.0 for some 130 magnetic observatories.

For reasons given by Campbell (1980) and Malin and Winch (1966), and for compatibility with Malin and Iş1kara (1976) we chose to analyse nighttime data, consisting of the mean of five hourly mean values centred on the hourly mean 
Table 1. Fourier coefficients for semi-annual variation (nighttime data). Unit: $0.01 \mathrm{nT}$. $\Phi$ denotes dip-latitude.

\begin{tabular}{|c|c|c|c|c|c|c|c|c|c|c|c|c|c|c|c|}
\hline \multirow{2}{*}{$\begin{array}{l}\text { Obs. } \\
\text { Code }\end{array}$} & \multirow{2}{*}{$\begin{array}{l}\text { Co- } \\
\text { lat. } \\
\left({ }^{\circ}\right)\end{array}$} & \multirow{2}{*}{$\begin{array}{c}\text { E. } \\
\text { long. } \\
\left(^{\circ}\right)\end{array}$} & \multirow{2}{*}{$\begin{array}{c}\Phi \\
\left(^{\circ}\right)\end{array}$} & \multicolumn{4}{|c|}{$X$} & \multicolumn{4}{|c|}{$Y$} & \multicolumn{4}{|c|}{$Z$} \\
\hline & & & & $a_{2}$ & $\sigma_{a}$ & $b_{2}$ & $\sigma_{b}$ & $a_{2}$ & $\sigma_{a}$ & $b_{2}$ & $\sigma_{b}$ & $a_{2}$ & $\sigma_{a}$ & $b_{2}$ & $\sigma_{b}$ \\
\hline ALE* & 7.5 & 297.5 & 82 & 552 & 79 & -141 & 139 & 566 & 62 & -564 & 54 & 802 & 102 & -593 & 107 \\
\hline HIS* & 9.4 & 58.0 & 79 & -61 & 107 & -139 & 95 & -50 & 129 & -931 & 78 & 630 & 257 & -639 & 196 \\
\hline $\mathrm{CCS}^{*}$ & 12.3 & 104.3 & 84 & -383 & 195 & -220 & 149 & -317 & 120 & -323 & 81 & 161 & 238 & -1475 & 322 \\
\hline THL* & 12.5 & 290.8 & 82 & -5 & 71 & -190 & 128 & -50 & 77 & -445 & 96 & 52 & 50 & -502 & 60 \\
\hline $\mathrm{MBC}^{*}$ & 13.8 & 240.6 & 85 & -391 & 98 & -195 & 91 & -140 & 95 & -128 & 113 & -36 & 99 & -946 & 77 \\
\hline RES$^{*}$ & 15.3 & 265.1 & 88 & -9 & 141 & -419 & 176 & -62 & 89 & -310 & 88 & 103 & 69 & -643 & 93 \\
\hline $\mathrm{DIK}^{*}$ & 16.4 & 80.6 & 78 & -1040 & 145 & 1447 & 262 & -212 & 186 & -93 & 222 & 300 & 126 & -1256 & 217 \\
\hline $\mathrm{TIK}^{*}$ & 18.4 & 129.0 & 76 & 76 & 175 & 1106 & 216 & -174 & 90 & -260 & 114 & -513 & 186 & -337 & 133 \\
\hline BRW* & 18.7 & 203.2 & 71 & -1062 & 512 & 1075 & 400 & -644 & 333 & -648 & 245 & -715 & 388 & -116 & 280 \\
\hline TRO* & 20.3 & 19.0 & 66 & -739 & 440 & 1303 & 479 & 182 & 94 & -369 & 117 & -181 & 286 & -454 & 211 \\
\hline $\mathrm{GDH}^{*}$ & 20.8 & 306.5 & 73 & -17 & 60 & -224 & 84 & -21 & 55 & -364 & 91 & -7 & 136 & -460 & 165 \\
\hline $\mathrm{MMK}^{*}$ & 21.8 & 33.1 & 65 & 248 & 240 & 1138 & 225 & -137 & 158 & -393 & 147 & -96 & 141 & 124 & 187 \\
\hline $\mathrm{KIR}^{*}$ & 22.2 & 20.4 & 66 & -522 & 672 & 690 & 447 & 48 & 185 & -202 & 174 & -677 & 306 & 194 & 337 \\
\hline SOD* & 22.6 & 26.6 & 65 & -112 & 542 & 1047 & 310 & -72 & 166 & -255 & 81 & -520 & 140 & 287 & 144 \\
\hline CWE* $^{*}$ & 23.8 & 190.2 & 62 & 47 & 116 & 324 & 150 & 185 & 51 & -136 & 49 & 278 & 105 & 27 & 105 \\
\hline $\mathrm{CMO}^{*}$ & 25.1 & 212.2 & 65 & 12 & 202 & 166 & 162 & 87 & 117 & -35 & 98 & 2 & 116 & 109 & 135 \\
\hline BLC* & 25.7 & 264.0 & 82 & -28 & 149 & 185 & 124 & -27 & 152 & -127 & 87 & 476 & 204 & -636 & 194 \\
\hline $\mathrm{LRV}^{*}$ & 25.8 & 338.3 & 64 & -513 & 514 & 1175 & 295 & 104 & 226 & -588 & 141 & -338 & 128 & 295 & 137 \\
\hline DOB & 27.9 & 9.1 & 60 & 59 & 122 & 279 & 96 & -40 & 78 & -177 & 64 & -21 & 187 & 463 & 170 \\
\hline NUR & 29.5 & 24.6 & 58 & 21 & 48 & 101 & 61 & -35 & 34 & -34 & 63 & -158 & 49 & 198 & 98 \\
\hline LER & 29.9 & 358.8 & 58 & 6 & 36 & 207 & 97 & 19 & 44 & -138 & 78 & -20 & 157 & 381 & 132 \\
\hline LNN & 30.0 & 30.7 & 58 & 12 & 36 & 114 & 48 & -78 & 27 & -97 & 62 & -34 & 43 & 152 & 74 \\
\hline LOV & 30.6 & 17.8 & 57 & 19 & 30 & 71 & 40 & -35 & 75 & -62 & 60 & -94 & 49 & 175 & 47 \\
\hline $\mathrm{FCC}^{*}$ & 31.2 & 265.9 & 77 & -431 & 221 & 588 & 179 & -1509 & 203 & -450 & 210 & 610 & 292 & -1036 & 193 \\
\hline SIT $^{*}$ & 32.9 & 224.7 & 60 & 72 & 70 & 164 & 138 & 81 & 52 & -144 & 93 & 41 & 89 & -54 & 124 \\
\hline SVD & 33.3 & 61.1 & 58 & -32 & 33 & 57 & 50 & -11 & 51 & -117 & 41 & -51 & 22 & -3 & 21 \\
\hline RSV & 34.2 & 12.4 & 54 & -37 & 37 & 142 & 51 & -45 & 38 & -83 & 69 & -53 & 47 & 204 & 49 \\
\hline KZN & 34.2 & 48.8 & 56 & -56 & 33 & 151 & 49 & 2 & 32 & -74 & 54 & 3 & 25 & 57 & 28 \\
\hline MOS & 34.5 & 37.3 & 55 & 32 & 29 & 138 & 29 & -48 & 40 & -59 & 45 & -45 & 13 & 44 & 17 \\
\hline ESK & 34.7 & 356.8 & 53 & -99 & 24 & 90 & 42 & -25 & 37 & -96 & 61 & -44 & 47 & 105 & 51 \\
\hline MEA* & 35.4 & 246.7 & 66 & 49 & 125 & 368 & 163 & 312 & 86 & 51 & 83 & -159 & 143 & 140 & 168 \\
\hline MNK & 35.9 & 26.5 & 53 & 87 & 37 & -68 & 30 & 28 & 44 & -76 & 26 & -11 & 22 & 14 & 25 \\
\hline STO & 36.2 & 357.5 & 52 & -72 & 33 & 14 & 32 & 39 & 38 & -124 & 28 & 11 & 28 & 70 & 25 \\
\hline WNG & 36.2 & 9.1 & 51 & 30 & 18 & -5 & 46 & -2 & 41 & -15 & 58 & -77 & 26 & 98 & 29 \\
\hline WIT & 37.2 & 6.7 & 51 & -56 & 34 & 106 & 43 & 47 & 31 & -34 & 60 & -32 & 25 & 46 & 18 \\
\hline IRT & 37.8 & 104.4 & 56 & 75 & 48 & 161 & 36 & 20 & 22 & -80 & 21 & -9 & 15 & 0 & 15 \\
\hline SWI & 37.9 & 21.2 & 51 & 46 & 39 & 84 & 41 & -4 & 39 & -58 & 45 & -396 & 34 & 123 & 24 \\
\hline NGK & 37.9 & 12.7 & 50 & 1 & 35 & 40 & 23 & -14 & 34 & -51 & 69 & -50 & 18 & 51 & 12 \\
\hline VAL & 38.1 & 349.8 & 50 & -45 & 34 & 165 & 38 & 37 & 32 & -95 & 53 & 45 & 39 & 101 & 27 \\
\hline HAD & 39.0 & 355.5 & 49 & 7 & 34 & 64 & 26 & 36 & 36 & -93 & 37 & -73 & 18 & 39 & 14 \\
\hline KIV & 39.3 & 30.3 & 49 & 96 & 35 & 170 & 33 & -83 & 23 & -118 & 36 & -6 & 11 & 189 & 16 \\
\hline DOU & 39.9 & 4.6 & 48 & 24 & 34 & 59 & 37 & -22 & 46 & -105 & 41 & -53 & 22 & 72 & 14 \\
\hline LVV & 40.1 & 23.8 & 48 & -34 & 32 & 52 & 42 & -19 & 37 & -75 & 33 & 38 & 21 & -5 & 24 \\
\hline VIC & 41.5 & 236.6 & 55 & -132 & 42 & 75 & 72 & 34 & 42 & -92 & 53 & 99 & 45 & -40 & 60 \\
\hline WIK & 41.7 & 16.3 & 46 & 30 & 36 & 106 & 35 & -7 & 45 & -66 & 52 & -65 & 13 & -14 & 12 \\
\hline FUR & 41.8 & 11.3 & 46 & 44 & 22 & 65 & 32 & 5 & 42 & -49 & 50 & -38 & 15 & 14 & 10 \\
\hline CLF & 42.0 & 2.3 & 46 & 157 & 73 & 77 & 25 & -88 & 43 & -78 & 59 & -237 & 28 & -269 & 22 \\
\hline HRB & 42.1 & 18.2 & 46 & -42 & 23 & 25 & 36 & 25 & 31 & -32 & 40 & 31 & 12 & -74 & 13 \\
\hline YSS & 43.0 & 142.7 & 41 & 131 & 71 & 118 & 52 & 13 & 25 & -73 & 19 & -75 & 19 & -183 & 24 \\
\hline THY & 43.1 & 17.9 & 45 & -60 & 64 & 153 & 30 & -59 & 51 & 29 & 56 & -161 & 26 & 85 & 27 \\
\hline MMB & 46.1 & 144.2 & 38 & 30 & 47 & 84 & 61 & 22 & 18 & -93 & 16 & -53 & 14 & -58 & 20 \\
\hline $\mathrm{AGN}^{*}$ & 46.2 & 280.7 & 60 & -49 & 66 & 259 & 87 & 17 & 34 & -50 & 37 & -172 & 77 & -341 & 64 \\
\hline VLA & 46.3 & 132.2 & 40 & 59 & 59 & 77 & 33 & 242 & 25 & -90 & 35 & 159 & 22 & -161 & 21 \\
\hline PAG & 47.5 & 24.2 & 40 & 27 & 51 & 121 & 17 & -19 & 15 & -18 & 39 & -80 & 14 & -86 & 10 \\
\hline AQU & 47.6 & 13.3 & 39 & 5 & 42 & 143 & 37 & -52 & 31 & -53 & 30 & -98 & 8 & -101 & 20 \\
\hline LGR & 47.6 & 357.5 & 39 & -63 & 20 & 37 & 23 & -12 & 31 & -38 & 40 & -66 & 17 & 7 & 21 \\
\hline TFS & 47.9 & 44.7 & 41 & 30 & 47 & 149 & 35 & 10 & 32 & -64 & 41 & -120 & 10 & -51 & 15 \\
\hline
\end{tabular}


Table 1. (continued)

\begin{tabular}{|c|c|c|c|c|c|c|c|c|c|c|c|c|c|c|c|}
\hline \multirow{2}{*}{$\begin{array}{l}\text { Obs. } \\
\text { Code }\end{array}$} & \multirow{2}{*}{$\begin{array}{l}\text { Co- } \\
\text { lat. } \\
\left({ }^{\circ}\right)\end{array}$} & \multirow{2}{*}{$\begin{array}{c}\text { E. } \\
\text { long. } \\
\left(^{\circ}\right)\end{array}$} & \multirow{2}{*}{$\begin{array}{c}\Phi \\
\left({ }^{\circ}\right)\end{array}$} & \multicolumn{4}{|c|}{$X$} & \multicolumn{4}{|c|}{$Y$} & \multicolumn{4}{|c|}{$Z$} \\
\hline & & & & $a_{2}$ & $\sigma_{a}$ & $b_{2}$ & $\sigma_{b}$ & $a_{2}$ & $\sigma_{a}$ & $b_{2}$ & $\sigma_{b}$ & $a_{2}$ & $\sigma_{a}$ & $b_{2}$ & $\sigma_{b}$ \\
\hline TKT & 48.6 & 69.2 & 41 & 20 & 41 & 148 & 51 & -81 & 39 & -11 & 28 & -33 & 18 & -22 & 13 \\
\hline EBR & 49.2 & 0.5 & 37 & -645 & 95 & 91 & 45 & 18 & 55 & -12 & 37 & -183 & 20 & 541 & 25 \\
\hline $\mathrm{COI}$ & 49.8 & 351.6 & 37 & -34 & 29 & 159 & 41 & -93 & 19 & -122 & 30 & 13 & 19 & -25 & 23 \\
\hline FRD & 50.1 & 282.6 & 54 & 28 & 43 & 221 & 52 & 44 & 28 & -38 & 29 & -33 & 22 & -54 & 15 \\
\hline TOL & 50.1 & 356.0 & 36 & -191 & 42 & 244 & 30 & 14 & 40 & -97 & 19 & -113 & 16 & 196 & 23 \\
\hline $\mathrm{ASH}$ & 52.0 & 58.1 & 37 & 215 & 38 & 165 & 76 & 57 & 31 & 32 & 24 & -191 & 15 & -38 & 15 \\
\hline ALM & 53.2 & 357.5 & 33 & 104 & 23 & 219 & 34 & 5 & 21 & -24 & 30 & -26 & 20 & -43 & 18 \\
\hline KAK & 53.8 & 140.2 & 30 & 99 & 60 & 118 & 47 & 35 & 17 & -78 & 19 & 15 & 20 & -54 & 20 \\
\hline TEH & 54.3 & 51.4 & 34 & -7 & 69 & 105 & 60 & 32 & 50 & -45 & 39 & -76 & 79 & 276 & 25 \\
\hline SSO & 56.4 & 135.9 & 28 & 19 & 78 & 109 & 58 & 37 & 16 & -77 & 14 & -54 & 17 & -48 & 30 \\
\hline DAL & 57.0 & 263.2 & 44 & 47 & 47 & 217 & 41 & 62 & 31 & 4 & 25 & -109 & 19 & -269 & 11 \\
\hline TUC & 57.8 & 249.2 & 40 & -55 & 55 & 197 & 39 & 81 & 21 & -2 & 26 & -50 & 9 & -57 & 13 \\
\hline KNY & 58.6 & 130.9 & 26 & 89 & 50 & 107 & 41 & -45 & 10 & -64 & 12 & -4 & 8 & -37 & 14 \\
\hline MLT & 60.5 & 30.9 & 25 & 91 & 58 & 176 & 47 & 82 & 19 & 40 & 17 & -204 & 10 & -107 & 8 \\
\hline HVN & 67.0 & 277.8 & 36 & 174 & 92 & 249 & 100 & -40 & 27 & -186 & 25 & -170 & 28 & -5 & 21 \\
\hline $\mathrm{HON}$ & 68.7 & 202.0 & 22 & 57 & 61 & 231 & 87 & 61 & 14 & 31 & 20 & 23 & 11 & 44 & 9 \\
\hline $\mathrm{ABG}$ & 71.4 & 72.9 & 13 & 159 & 58 & -18 & 49 & 105 & 16 & -55 & 20 & -234 & 33 & -819 & 47 \\
\hline SJG & 71.9 & 293.8 & 32 & -113 & 41 & 330 & 48 & 264 & 14 & -20 & 16 & -71 & 14 & -22 & 12 \\
\hline HYB & 72.6 & 78.6 & 11 & 105 & 131 & 94 & 98 & -22 & 58 & 106 & 89 & 130 & 52 & -224 & 41 \\
\hline MBO & 75.6 & 343.0 & 8 & -51 & 51 & 289 & 52 & 5 & 18 & 57 & 20 & -181 & 11 & -219 & 10 \\
\hline GUA & 76.4 & 144.9 & 6 & 48 & 27 & 154 & 74 & -19 & 6 & -26 & 12 & -87 & 22 & 23 & 25 \\
\hline ANN & 78.6 & 79.7 & 3 & -161 & 87 & -68 & 72 & -346 & 36 & 286 & 71 & -174 & 26 & -96 & 31 \\
\hline AAE & 81.0 & 38.8 & -1 & 101 & 42 & 190 & 32 & -151 & 33 & -262 & 33 & 189 & 31 & 102 & 17 \\
\hline KOR & 82.7 & 134.5 & 0 & -351 & 225 & -3 & 218 & -59 & 25 & -158 & 15 & -472 & 108 & -420 & 58 \\
\hline PAB & 84.2 & 304.8 & 17 & -4 & 46 & 352 & 40 & 98 & 17 & -84 & 17 & -141 & 10 & -39 & 19 \\
\hline FUQ & 84.5 & 286.3 & 18 & 120 & 72 & 93 & 45 & -47 & 17 & -47 & 13 & -224 & 25 & -199 & 40 \\
\hline BNG & 85.6 & 18.6 & -7 & 231 & 49 & 533 & 66 & -21 & 14 & -352 & 20 & 95 & 18 & 44 & 25 \\
\hline MFP & 86.6 & 8.7 & -8 & 85 & 54 & 163 & 42 & -186 & 20 & 27 & 22 & -34 & 11 & -32 & 9 \\
\hline TTB & 91.2 & 311.5 & 8 & 4 & 88 & 308 & 46 & -32 & 30 & -29 & 28 & -76 & 31 & -53 & 18 \\
\hline LWI & 92.3 & 28.8 & -16 & -329 & 104 & 143 & 67 & -36 & 19 & 37 & 20 & -21 & 5 & -64 & 9 \\
\hline PMG & 99.4 & 147.2 & -18 & -44 & 33 & 190 & 41 & 30 & 17 & -96 & 20 & 63 & 16 & -120 & 16 \\
\hline HUA & 102.0 & 284.7 & 1 & 68 & 62 & 491 & 39 & 59 & 19 & 21 & 15 & 5 & 15 & -8 & 12 \\
\hline API & 103.8 & 188.2 & -16 & -145 & 72 & 111 & 59 & -155 & 21 & -46 & 26 & 81 & 14 & 55 & 17 \\
\hline TAN & 108.9 & 47.6 & -35 & 18 & 43 & 222 & 52 & -91 & 13 & 45 & 24 & 17 & 16 & 78 & 28 \\
\hline TSU & 109.2 & 17.6 & -38 & -95 & 69 & 318 & 84 & -39 & 27 & -57 & 26 & -11 & 24 & 81 & 23 \\
\hline PIL & 121.7 & 296.1 & -15 & -458 & 64 & 125 & 65 & -41 & 21 & -30 & 19 & -255 & 22 & 77 & 55 \\
\hline GNA & 121.8 & 116.0 & -48 & 31 & 37 & 96 & 61 & -22 & 27 & -66 & 21 & 13 & 14 & -38 & 14 \\
\hline HER & 124.4 & 19.2 & -47 & -14 & 51 & 179 & 68 & -18 & 27 & 21 & 38 & 8 & 21 & -18 & 28 \\
\hline TOO & 127.5 & 145.5 & -51 & -10 & 48 & 77 & 38 & 39 & 40 & -92 & 31 & -19 & 21 & 46 & 24 \\
\hline AML & 133.2 & 172.7 & -51 & 257 & 68 & 150 & 55 & 187 & 48 & -106 & 49 & 172 & 47 & 38 & 29 \\
\hline TRW & 133.2 & 294.7 & -22 & 49 & 99 & -122 & 73 & -64 & 55 & -94 & 42 & -248 & 90 & -92 & 28 \\
\hline KGL & 139.4 & 70.2 & -50 & -211 & 48 & 171 & 45 & -86 & 46 & 167 & 45 & 51 & 26 & -62 & 34 \\
\hline $\mathrm{MCQ}^{*}$ & 144.5 & 159.0 & -68 & -212 & 304 & 1027 & 185 & 207 & 160 & 244 & 100 & 120 & 137 & -403 & 73 \\
\hline AIA & 155.2 & 295.7 & -38 & 30 & 37 & 189 & 35 & 2 & 30 & 56 & 32 & 110 & 26 & -66 & 40 \\
\hline MIR* $^{*}$ & 156.6 & 93.0 & -65 & -125 & 157 & 364 & 76 & 59 & 116 & -43 & 154 & -18 & 123 & 1044 & 120 \\
\hline $\mathrm{DRV}^{*}$ & 156.7 & 140.0 & -89 & 233 & 69 & 49 & 54 & 2 & 70 & 554 & 39 & -689 & 178 & 1245 & 155 \\
\hline MAW & 157.6 & 62.9 & -53 & -234 & 238 & 1113 & 247 & -100 & 338 & -1161 & 408 & 354 & 196 & 347 & 158 \\
\hline $\mathrm{RBD}$ & 160.4 & 24.3 & -47 & 107 & 249 & 457 & 140 & -255 & 185 & -376 & 110 & 354 & 268 & -410 & 200 \\
\hline SNA & 160.5 & 357.5 & -45 & -97 & 64 & 359 & 78 & 321 & 51 & -210 & 59 & 430 & 90 & -37 & 55 \\
\hline NVL & 160.8 & 11.8 & -46 & 127 & 138 & 791 & 97 & 548 & 101 & 346 & 72 & 76 & 61 & -445 & 116 \\
\hline EGS & 165.2 & 282.8 & -49 & 76 & 105 & 123 & 103 & 131 & 86 & -4 & 72 & 519 & 162 & -84 & 216 \\
\hline HBA & 165.5 & 333.4 & -47 & 109 & 291 & 895 & 180 & 210 & 156 & 175 & 85 & 357 & 327 & -584 & 151 \\
\hline SBA* $^{*}$ & 167.2 & 166.8 & -74 & -79 & 137 & -246 & 137 & 112 & 42 & 162 & 35 & -187 & 101 & 1284 & 117 \\
\hline VOS* & 168.4 & 106.9 & -68 & 1956 & 121 & -668 & 157 & -1707 & 102 & 895 & 145 & -7 & 97 & 1025 & 130 \\
\hline $\mathrm{BYR}^{*}$ & 170.0 & 240.0 & -61 & -87 & 154 & 452 & 196 & -20 & 385 & 589 & 497 & -266 & 278 & 819 & 260 \\
\hline SPA* $^{*}$ & 180.0 & 0.0 & -61 & 74 & 160 & -80 & 124 & 971 & 152 & -134 & 129 & 425 & 151 & 1820 & 151 \\
\hline
\end{tabular}

*Not used in the spherical harmonic analysis. 


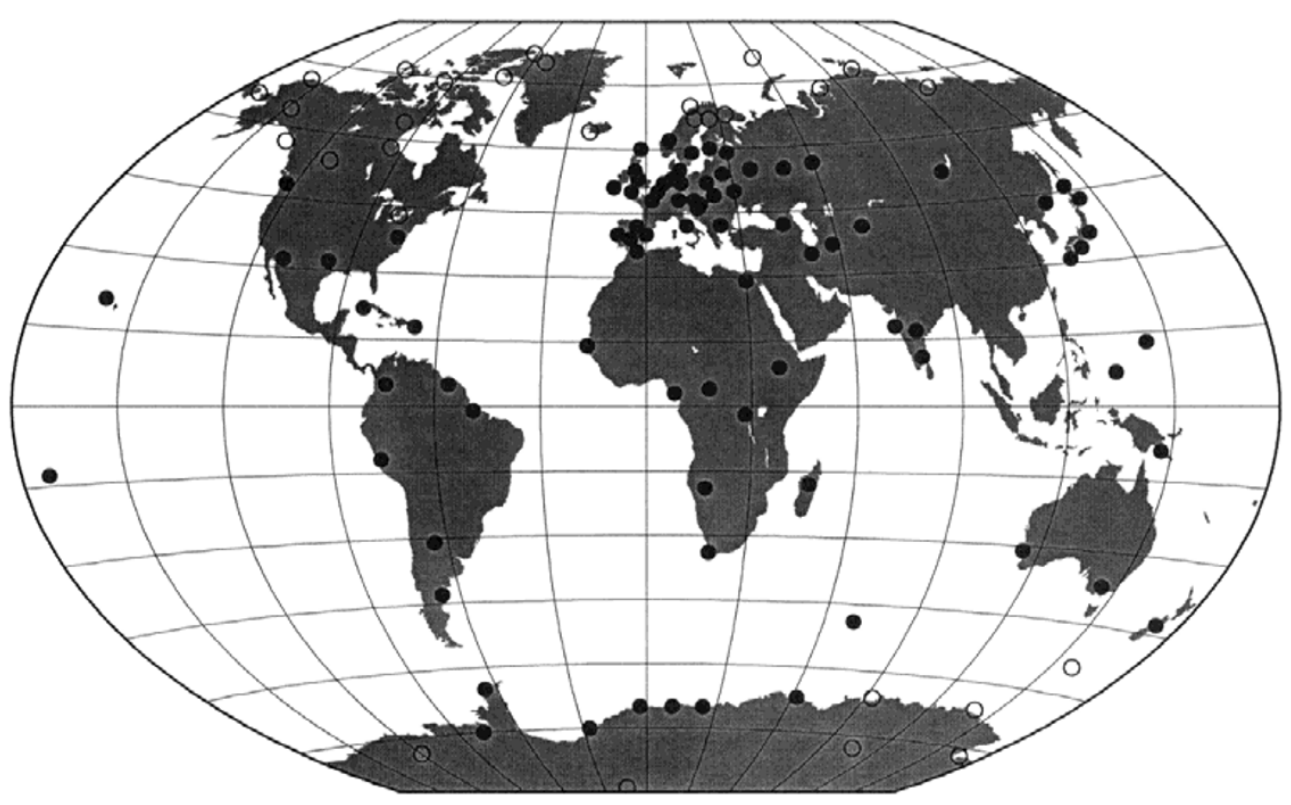

Fig. 1. Locations of the observatories listed in Table 1. Those marked by open circles are omitted from the spherical harmonic analysis.

value that includes local midnight, one mean for each day. These we call the "nighttime means".

\section{Method of Analysis}

This is closely similar to that described by Malin and Schlapp (1980). For a given observatory, each nighttime mean provides an equation of condition of the form

$H_{(t)}=H_{0}+a_{0} t+a_{1} \cos t+b_{1} \sin t+a_{2} \cos 2 t+b_{2} \sin 2 t$,

where $t$ increases from 0 to $2 \pi$ from January 0.0 to December $31.0, H_{0}$ represents a constant term, $a_{0} t$ the secular variation (assumed to be linear over the 2-year interval analysed), $a_{1} \cos t+b_{1} \sin t$ the annual variation and $a_{2} \cos 2 t+b_{2} \sin 2 t$ the semi-annual variation.

The constants $H_{0}, a_{0}, a_{1}, b_{1}, a_{2}, b_{2}$, were determined, separately for each observatory and element, from the equations of condition by least-squares analysis of all the data. Their standard deviations were obtained as detailed by Malin et al. (1996). We denote those for $a_{2}$ and $b_{2}$ by $\sigma_{a}$ and $\sigma_{b}$ respectively. The values of $a_{2}, b_{2}, \sigma_{a}$ and $\sigma_{b}$ are the data for subsequent global analysis of the semi-annual variation.

All the $H$ and $D$ results were converted to $X$ and $Y$.

\section{Quality Control}

The nighttime means were plotted, together with curves synthesized from $\left(H_{0}, a_{0}\right)$ and $\left(H_{0}, a_{0}, a_{1}, b_{1}, a_{2}, b_{2}\right)$. Each of these plots was examined for implausible values and for discontinuities, and these were checked against the original sources. Most of the apparently wild values were found to be real, but a few typographical errors (in the data for Pilar and Teoloyucan) were detected and corrected. The discontinuities resulted from unexplained changes of baseline. High quality baseline control is required for the reliable determination of the semi-annual term. Several of the observatories (particularly whose primary purpose was the monitoring of shorter-period variations) either did not monitor their baselines, or had inadequate documentation of them. These observatories were rejected.

The results are given in Table 1, with the observatories listed in order of colatitude. Their geographic positions are shown in Fig. 1.

\section{Spherical Harmonic Analysis}

We represent the global-scale features of the semi-annual variation with a spherical harmonic model of modest order and degree. Such a model would be expected to represent those parts of the variation that originate in the ring-current and magnetosphere, together with the induced reflection of these within the Earth. It could not hope to reproduce the high-latitude effects (ascribed by Malin and Iş1kara (1976) to the auroral electrojets) where large fluctuations occur over a small range of latitude. Neither could it model any oceancorrelated effect, since the oceanic outlines require spherical harmonics of higher order and degree for their representation.

To avoid swamping the model with the large auroral-latitude variations, we followed the example of Malin and Iş1kara (1976) in excluding from the analysis all observatories at high dip-latitude $\left(|\Phi|>60^{\circ}\right.$, where $\tan \Phi=\frac{1}{2} \tan I_{\mathrm{m}}=$ $Z_{\mathrm{m}} / 2 H_{\mathrm{m}}$; and $I_{\mathrm{m}}, Z_{\mathrm{m}}$ and $H_{\mathrm{m}}$ denote the observatory values of dip, vertical and horizontal intensity, respectively, meaned over the data-interval). There were 29 such observatories. This left 84 observatories with $X, Y$ and $Z$ data suitable for spherical harmonic analysis, giving two sets of 252 equations of condition, one set for $a_{2}$ and one for $b_{2}$. The equations were weighted inversely as the variance of $a_{2}$ (or $b_{2}$ ) and solved by the method of least squares, using matrix inversion. The standard deviation of the spherical harmonic coefficients are given by $s d=(Q W / N)^{1 / 2}$, where $Q$ denotes the sum of squares of weighted residuals, $W$ is the diagonal element of the inverse matrix corresponding to the required spherical harmonic and $N$ denotes the number of degrees of freedom. 
Table 2. Spherical harmonic coefficients of the semi-annual variation, including only harmonics with amplitudes significantly different from zero at the $95 \%$ level. The units are $\mathrm{pT}$.

\begin{tabular}{|c|c|c|c|c|c|}
\hline & \multicolumn{2}{|c|}{ Internal part } & \multicolumn{2}{|c|}{ External part } & \multirow[b]{2}{*}{$A I C$} \\
\hline & $a_{2}$ & $b_{2}$ & $a_{2}$ & $b_{2}$ & \\
\hline \multicolumn{5}{|c|}{ Analysis for the optimum number of coefficients } & \\
\hline$g_{1}^{0}$ & $204 \pm 95$ & $-544 \pm 111$ & $-615 \pm 178$ & $-1836 \pm 207$ & \\
\hline$g_{1}^{1}$ & & & $-510 \pm 86$ & $-153 \pm 106$ & \\
\hline$g_{2}^{0}$ & & & $192 \pm 87$ & $542 \pm 107$ & 426.32 \\
\hline$g_{2}^{2}$ & $-137 \pm 45$ & $-108 \pm 55$ & $23 \pm 53$ & $244 \pm 68$ & \\
\hline$g_{3}^{3}$ & & & $27 \pm 46$ & $-297 \pm 62$ & \\
\hline$g_{4}^{1}$ & $-142 \pm 45$ & $-206 \pm 49$ & & & \\
\hline
\end{tabular}

Analysis for the optimum number of coefficients, external and internal coefficients

$\begin{array}{rrccr}g_{1}^{0} & 330 \pm 112 & -470 \pm 131 & -432 \pm 208 & -1832 \pm 239 \\ g_{1}^{1} & 86 \pm 60 & -99 \pm 73 & -385 \pm 102 & -182 \pm 120 \\ g_{2}^{0} & -243 \pm 87 & -114 \pm 107 & -94 \pm 123 & 376 \pm 148 \\ g_{2}^{2} & -162 \pm 47 & -83 \pm 58 & 6 \pm 57 & 250 \pm 73 \\ g_{3}^{3} & -52 \pm 41 & 24 \pm 53 & -1 \pm 48 & -280 \pm 63 \\ g_{4}^{1} & -25 \pm 57 & -154 \pm 64 & 169 \pm 68 & 131 \pm 76\end{array}$

Analysis for the $g_{1}^{0}$ (dipole) term only

$g_{1}^{0} \quad 273 \pm 88 \quad-533 \pm 105$

$-333 \pm 161 \quad-1287 \pm 191$

451.48

Separate analyses are performed for $a_{2}$ and $b_{2}$, though both are part of the same harmonic. For this reason we choose to determine the same set of spherical harmonic coefficients for $a_{2}$ as for $b_{2}$. We judge the significance of a spherical harmonic term by $R$, the ratio of its amplitude to its vector standard deviation,

$$
R=\left(A^{2}+B^{2}\right)^{1 / 2} /\left(\sigma_{A}^{2}+\sigma_{B}^{2}\right)^{1 / 2} .
$$

Here, $A$ and $B$ denote corresponding spherical harmonic coefficients from the $a_{2}$ and $b_{2}$ analyses respectively, and $\sigma_{A}$ and $\sigma_{B}$ denote their standard deviations. If $R$ exceeds 1.73 the harmonic is significantly different from zero at the 5 per cent level.

Which spherical harmonic coefficients should be included in the model? Tradition dictates that the model should be complete up to some chosen order and degree, but this is merely a hangover from the days of hand-computation and is no longer necessary. The larger the number of coefficients, the smaller will be the sum of squares of residuals and the closer the fit to the data. But closeness of fit is not a satisfactory criterion. When the number of coefficients equals the number of observations an exact, but not necessarily realistic, fit will be obtained.

Following Anderssen (1969), we consider the ideal model to be that that includes all the harmonics that are significant, and none that are not. Unfortunately, this does not define a unique model, since the significance of a coefficient depends on the set of other coefficients with which it is determined. We have found different, but self-consistent, sets of coefficients that satisfy the above criterion experimentally, and for different significance levels. In such cases, we opt for the model with the smaller standard deviations.

Our strategy to find a model that satisfies the requirements is as follows.

1. Choose a level of significance, e.g. $R>1.73$ for the 95 per cent level, or $R>1$ if we merely wish the harmonic to exceed its vector standard deviation (see Malin and Schlapp, 1980, Appendix).

2. Supply an initial set of harmonics. This can be the null set, but in our opinion it is preferable to do a few pilot analyses starting with a full set of harmonics and, for each successive analysis, dropping those that were not significantly determined. After a very few such iterations, all the remaining coefficients are significant, and this set of harmonics forms a suitable "initial set".

3. Up to a chosen upper limit, add the missing harmonics one at a time and re-analyse. If one of the added harmonics should yield a significant coefficient, it is included in the new "initial set" and the process starts again. Any harmonic that is insignificant in the presence of the new one is omitted.

When the process is complete, all the chosen coefficients 


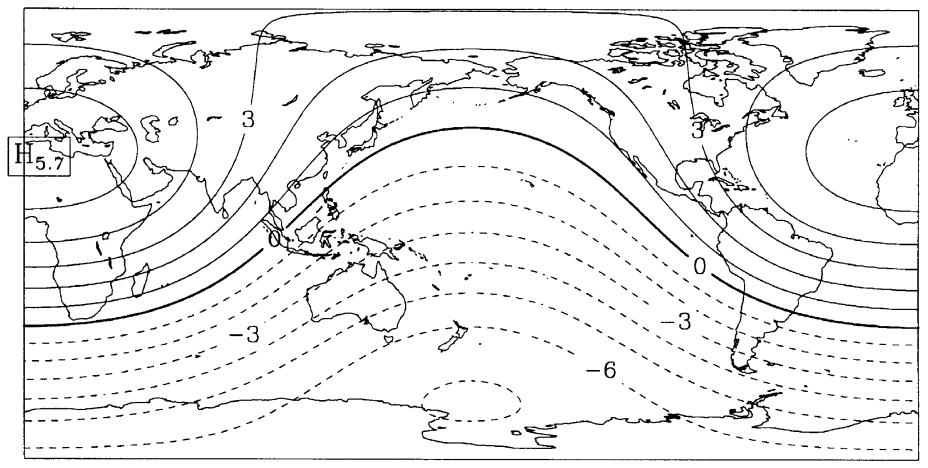

External current function 2 cpa A coeffs, kA computed from midnight values

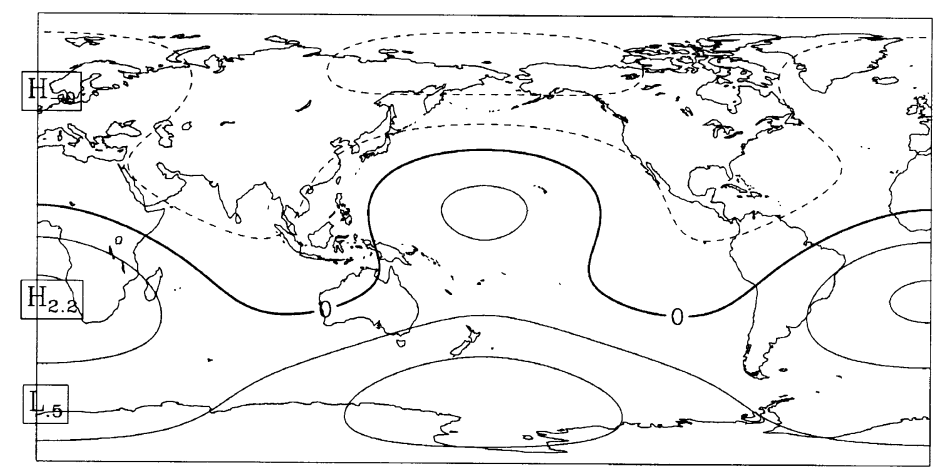

Internal current function 2 cpa A coeffs, $\mathrm{kA}$ computed from midnight values

Fig. 2. The internal and external equivalent current systems for the semi-annual variation at January 0 (or July 2). Currents flow clockwise around positive contours, anticlockwise around negative; the units are kA. For April 1 (or October 1) the currents are reversed.

are significant, and no single one of those omitted would be significant if it were included with the final set.

We have arbitrarily chosen an upper limit of $m=n=4$ for both the internal and external parts, giving a total of 48 available spherical harmonics. Here $m$ denotes the order and $n$ the degree of the spherical harmonic. It was not considered that the data were adequate in quality or distribution for the determination of higher order and degree coefficients.

\section{Results and Discussion}

Table 2 gives the results of the analysis for the optimal model with only 6 spherical harmonics, there being 5 external and 3 internal coefficients for and for $a_{2}$ and for $b_{2}$. Also tabulated are the results when all 6 internal and external terms for $a_{2}$ and for $b_{2}$ are included, and given in the last row of Table 2 is the result for a model consisting of a dipole term only. Curiously, all the harmonics are $g_{n}^{m}$ terms, being the coefficients of $P_{n}^{m} \cos m \lambda$ where $\lambda$ denotes east longitude. None of the $h_{n}^{m}$ terms (the coefficient of $P_{n}^{m} \sin m \lambda$ ) differs significantly from zero. The dominant harmonic is external $g_{1}^{0}$. The ratio of the internal to the external part of $g_{1}^{0}$ is $0.300 \pm 0.064$. This is unexpectedly large compared with $0.105 \pm 0.032$ found by Malin and Iş1kara (1976) for the annual term and $0.12 \pm 0.07$ for the 11 -year variation (Harwood and Malin, 1977). It is closer to the ratio 0.37 found for the daily (24 hour) variation (Malin, 1973).

To show the significance of the chosen spherical harmonics in the potential for semi-annual variation, the Akaike Information Criterion (AIC), as defined by Akaike (1974) can be used, with $N$ as the number of data points,

$$
\begin{aligned}
A I C= & N \log (\text { mean square residual }) \\
& +2 \text { (number of parameters }) .
\end{aligned}
$$

For the chosen model with 16 spherical harmonic coefficients, the sum of squares of residuals is 6541.73 and the $A I C$ is 426.32 . Other converged models, typically with 24 spherical harmonic coefficients, have a smaller sum of squares of residuals 6064.73, but a larger $A I C$ of 452.78 . The increased number of spherical harmonic coefficients gives a reduction in the sum of squares of residuals but an overall increase in the $A I C$. The model given in Table 1 has the smallest $A I C$ of all converged models, as required for a preferred model.

The equivalent internal and external current functions associated with the model are shown in Figs. 2 and 3. Figure 2 shows the currents deduced from the analysis of $a_{2}$ which correspond to those flowing at the start and middle of the 


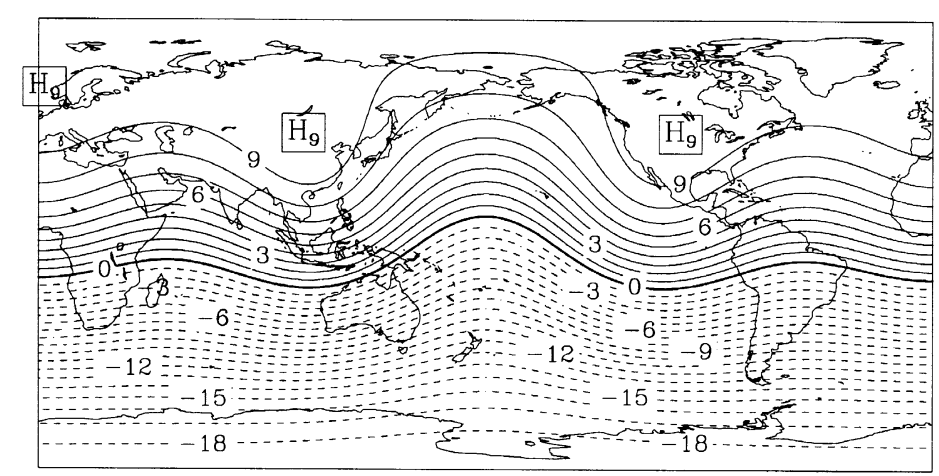

External current function 2 cpa B coeffs, kA computed from midnight values

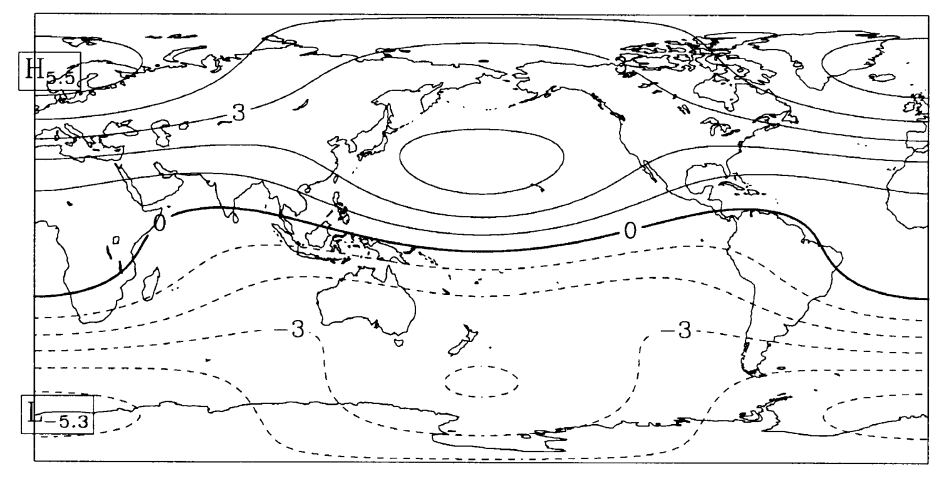

Internal current function 2 cpa B coeffs, $k A$ computed from midnight values

Fig. 3. The internal and external equivalent current systems for the semi-annual variation at February 15 (or August 16). Currents flow clockwise around positive contours, anticlockwise around negative; the units are kA. For May 17 (or November 16) the currents are reversed.

year. Figure 3, from the $b_{2}$ coefficients, represents the currents one eighth or five eighths through the year (February 15 or August 16). The currents are represented by flow parallel to the contours in a (hypothetical) thin spherical shell concentric with and of the same radius as the Earth. By far the largest currents are the external ones. The internal currents show interesting features over the Pacific Ocean, but this is probably because of the paucity of the data there, rather than a real effect. Note however, that the coefficients in the preferred model are all statistically significant at the 95\% level, and every effort has been made to minimise the degree $n$ of the analysis and the number of terms. The exclusion of high-latitude observatories has further reduced noise in the input.

Considering only the $P_{1}^{0}$ external coefficient, the maximum semi-annual variation is found to have a 95 per cent probability of occurring between January 30 and February 11. This is closer to the March 7 time predicted by the heliographic latitude hypothesis than to March 21 time of the equinoctial hypothesis, but is uncomfortably earlier than both predictions. For $P_{2}^{0}$, external, the extremum (this time a minimum) has a 95 per cent probability of occurring between February 21 and March 1, again favouring the heliographic latitude hypothesis over the equinoctial.

It is common when determining $g_{1}^{0}$ for induction studies to do an analysis that includes no other terms. Since the observatories are far from being orthogonally distributed, the determined coefficients $g_{1}^{0}$ will contain a contribution from the undetermined harmonics. To see how serious this effect might be on the internal/external ratio we have done such an analysis. The results are given in the final row of Table 2 . The greatest change is to the dominant term, for $b_{2}$ external, which is diminished by 30 per cent. The ratio of external to internal amplitude is $0.45 \pm 0.10$. Clearly, seriously misleading ratios can be obtained from such single term harmonic analyses, and this is likely to apply to frequencies other than the semiannual.

Acknowledgments. The authors would like to thank the referees Professor Masaki Matsushima and Dr. Roger Banks for their comments and constructive criticism. The research was supported by Australian Research Committee Large Grant A69702642.

\section{References}

Akaike, H., A new look at the statistical model identification, IEEE Trans. Autom. Contr., AC-19, 716-723, 1974.

Anderssen, R. S., On the solutions of certain overdetermined systems of 
linear equations that arise in geophysics, J. Geophys. Res., 74, 1045$1051,1969$.

Banks, R. J., The overall conductivity distribution of the earth, J. Geomag. Geoelectr., 24, 337-351, 1972.

Black, D. I., Lunar and solar magnetic variations at Abinger: their detection and estimation by spectral analysis via Fourier transformation, Phil. Trans. R. Soc. London, A268, 233-263, 1970.

Butcher, E. C. and D. M. Schlapp, The annual variation of the night-time values of the geomagnetic field, Geophys. J. Int., 111, 151-158, 1992.

Campbell, W. H., Secular, annual, and semi-annual changes in the baseline level of the Earth's magnetic field at North American locations, $J$. Geophys. Res., 85, 6557-6571, 1980.

Campbell, W. H., Annual and semiannual variations of the geomagnetic field at equatorial locations, J. Atmos. Terr. Phys., 43, 607-616, 1981.

Fukushima, N. and T. Nagata, Morphology of magnetic disturbance, Ann. Geophys., 24, 1-11, 1968.

Gupta, J. C. and S. Chapman, Some statistics concerning the daily geomagnetic character figure Cp, Pure Appl. Geophys., 68, 103-113, 1967,

Harwood, J. M. and S. R. C. Malin, Sunspot cycle influence on the geomagnetic field, Geophys. J. R. Astr. Soc., 50, 605-619, 1977.

Hibberd, F. M., The geomagnetic $S q$ variation-annual, semiannual and solar cycle variations and ring current effects, J. Atmos. Terr. Phys., 47, 341-352, 1985.

Malin, S. R. C., Worldwide distribution of geomagnetic tides, Phil. Trans. R. Soc. London, A274, 551-594, 1973.

Malin, S. R. C. and A. M. Işıkara, Annual variation of the geomagnetic field, Geophys. J. R. Astr. Soc., 47, 445-457, 1976.

Malin, S. R. C. and D. M. Schlapp, Geomagnetic lunar analysis by least squares, Geophys. J. R. Astr. Soc., 60, 409-418, 1980.

Malin, S. R. C. and D. E. Winch, Annual variation of the geomagnetic field, Geophys. J. Int., 124, 170-174, 1996.

Malin, S. R. C., M. K. Tunçer, and O. Yazici-Çakim, Systematic analysis of magnetic observatory data-1. A proposed method, Geophys. J. Int., 126, 635-644, 1996.

McCreadie, H. and E. C. Butcher, Preliminary analysis of annual and semiannual variations of the geomagnetic field, Explor. Geophys., 24, 151156, 1993.

McIntosh, D. H., On the annual variation of magnetic disturbance, Phil. Trans. R. Soc. London, A251, 525-552, 1959.

McLeod, M. G., Magnetospheric and ionospheric signals in magnetic observatory monthly means: electrical conductivity of the deep mantle, $J$. Geophys. Res., 99, 13577-13590, 1994.

Rangarajan, G. K. and B. N. Bhargava, Long term changes in the amplitude and phase of the semiannual and annual variations in low latgitude geomagnetic field, J. Geomag. Geoelectr., 39, 437-446, 1987.

Rastogi, R. G., Geomagnetic field variations at low latitudes and ionospheric electric fields, J. Atmos. Terr. Phys., 55, 1375-1381, 1993.

Rastogi, R. G., S. Alex, and A. Patil, Seasonal variations of geomagnetic $D, H$ and $Z$ fields at low latitudes, J. Geomag. Geoelectr., 46, 115-126, 1994.

Russell, C. T. and R. L. McPherron, Semi-annual variation of geomagnetic activity, J. Geophys. Res., 78, 92-108, 1973.

S. R. C. Malin (e-mail: malin@boun.edu.tr), D. E. Winch, and A. M. Işıkara 